\title{
1 Environmental stress determines the colonization and impact of an endophytic fungus
}

\section{2 on invasive knotweed}

3

4 Sigisfredo Garnica ${ }^{1}$, Zhiyong Liao ${ }^{2,3}$, Samuel Hamard ${ }^{2,4}$, Frank Waller ${ }^{5}$, Madalin Parepa ${ }^{2}$,

5 Oliver Bossdorf ${ }^{2}$

6

$7 \quad{ }^{1}$ Instituto de Bioquímica y Microbiología, Facultad de Ciencias, Universidad Austral de

8 Chile, Valdivia, Chile

$9{ }^{2}$ Plant Evolutionary Ecology, Institute of Evolution \& Ecology, University of Tübingen,

10 Germany

$11{ }^{3}$ CAS Key Laboratory of Tropical Forest Ecology, Xishuangbanna Tropical Botanical

12 Garden, Chinese Academy of Sciences, Mengla, China

$13{ }^{4}$ Laboratoire Ecologie Fonctionnelle et Environnement, Université de Toulouse, France

$14{ }^{5}$ Pharmaceutical Biology, Julius Sachs Institute for Biosciences, University of Würzburg,

15 Germany

16

17 Correspondence: Oliver Bossdorf, Tel. +49 707129 78809, oliver.bossdorf@uni-tuebingen.de 


\section{Abstract}

1. There is increasing evidence that microbes play a key role in some plant invasions. A diverse and widespread but little understood group of plant-associated microbes are the fungal root endophytes of the order Sebacinales. They are associated with exotic populations of invasive knotweed (Reynoutria ssp.) in Europe, but their effects on the invaders are unknown.

2. We used the recently isolated Sebacinales root endophyte Serendipita herbamans to experimentally inoculate invasive knotweed and study root colonisation and effects on knotweed growth under different environmental conditions. We verified the inoculation success and fungal colonisation through immunofluorescence microscopy and qPCR.

3. We found that $S$. herbamans strongly colonized invasive knotweed in low-nutrient and shade environments, but much less under drought or benign conditions. At low nutrients, the endophyte had a positive effect on plant growth, whereas the opposite was true under shaded conditions.

4. Synthesis. Our study demonstrates that the root endophyte $S$. herbamans has the potential to colonize invasive knotweed fine roots and impact its growth, and it could thus also play a role in natural populations. Our results also show that effects of fungal endophytes on plants can be strongly environment-dependent, and may only be visible under stressful environmental conditions.

\section{Key words}

Biological invasions, drought, fungal endophytes, Japanese knotweed, nutrient availability, plant-microbe interactions, Reynoutria japonica, Sebacinales, shading, stress tolerance 


\section{Introduction}

44 Fungal endophytes are a phylogenetically diverse and widespread group of plant-associated microbes (Rodriguez et al., 2009). They can influence the growth and reproduction of individual plants, or their resistance to abiotic stress or natural enemies (Cosme et al., 2016;

Kivlin et al., 2013; Mayerhofer et al., 2013; Oberhofer et al., 2014; Rho et al., 2018;

Rodriguez et al., 2008). Some of the positive effects are related to the ability of endophytes to improve the nutrition of their host plants (Behie \& Bidochka, 2014). There is also evidence that endophytes can influence the diversity and composition of entire plant communities (Afkhami \& Strauss, 2016; Aguilar-Trigueros \& Rillig, 2016; Clay \& Holah, 1999; Rudgers et al., 2004; Rudgers et al., 2005) as well as their associated ecological networks (e.g. herbivores and their parasitoids; Omacini et al., 2001). However, so far our understanding of fungal endophytes is based on experiments with very few taxa, in particular the genus Neotyphodium and its asexual stage Epichlö̈: Other fungal systems have been hardly studied, mainly because most fungal endophytes are often difficult to cultivate and thus controlled experiments for testing their ecological functions have so far been impossible. An important group of fungal endophytes for which this has long been true is the Serendipitaceae family in the order of Sebacinales that contains many species with broad geographic and host ranges (Garnica et al., 2016; Weiss et al., 2011). Previous experimental work has so far been largely restricted to Serendipita indica (Piriformospora indica), and it showed that $P$. indica stimulates plant growth and influences plant nutrition and tolerances to biotic and abiotic stresses (Achatz et al., 2010; Barazani et al., 2005; Gill et al., 2016; Waller et al., 2005). Our group in Tu $\square$ bingen recently isolated and cultivated another widespread Serendipitaceae species, Serendipita herbamans, which is abundant and associated with a broad range of host species and habitats in Central Europe (Riess et al., 2014). 
67 Soil microbes can influence plant growth and stress tolerance, and these effects are to

68 some extent host plant-specific. As a consequence, plant-microbe interactions play a role in

69 structuring plant communities, and there is increasing evidence that they are also important in

70 the invasion of exotic plant species (Callaway et al., 2004; Dawson \& Schrama, 2016; Inderjit

71 \& van der Putten, 2010; Klironomos, 2002). In general, plant-associated microbes may have

72 positive or negative feedbacks on plants (Bever et al., 2012; van der Putten et al., 2013). If

73 exotic plants accumulate biota with overall more positive effects, maybe because some of the

74 their native pathogens did not make it to the introduced range, this may give invaders an

75 advantage over native plants (Callaway et al., 2011; Maron et al., 2014; Mitchell \& Power,

76 2003; Reinhart et al., 2003). Alternatively, exotic plants may influence soil biota to the

77 detriment of the native plants, e.g. through increasing abundances of their pathogens (Mangla

78 \& Callaway, 2007) or disrupting interactions with mutualists (Meinhardt \& Gehring, 2012;

79 Stinson et al., 2006).

80 Most previous research on plant-microbe interactions and plant invasion has focused on

81 soil-borne microbes rather than endophytes, even though fungal endophytes are clearly

82 abundant and diverse also in invasive plant populations (Clay et al., 2016; Shipunov et al.,

83 2008). Besides an interesting series of studies by Aschehoug et al. (2012, 2014) who

84 demonstrated that the leaf endophyte Alternaria alternata makes invasive knapweed

85 (Centaurea stoebe) more competitive and allelopathic against native North American grasses,

86 there has so far been little experimental work on fungal endophytes and invasive plants.

87 One of the most problematic plant invaders of temperate Europe and North America is

88 the Japanese knotweed (Reynoutria japonica) and its hybrid $R . \times$ bohemica. Their aggressive

89 growth can damage buildings and other structures, and it has huge impacts on native plant

90 communities and ecosystems (Aguilera et al., 2010; Gerber et al., 2008; Hejda et al., 2009).

91 Because of these ecological and economic costs, there is great interest in controlling invasive 
92 knotweed, and in understanding the biological mechanisms contributing to its success.

93 Previous experimental research indicates that chemical or microbial processes belowground,

94 or their interplay, may contribute to knotweed invasion success (Murrell et al., 2011; Parepa

et al., 2013; Siemens \& Blossey, 2007). However, the precise mechanisms and in particular

microbial taxa involved in these phenomena are unknown.

In a preliminary screening of some invasive knotweed populations around Tübingen

(see Supplementary Information) we had found a large diversity of root-associated fungi, and

$40 \%$ of the studied fine-root samples also harboured ITS sequences of Sebacinales fungi,

including S. herbamans (Table S1). Thus, interactions between invasive knotweed and

Sebacinales appeared to be common, and we were curious about the nature of the interaction

that if $S$. herbamans had an effect on knotweed, it would depend on environmental conditions,

in particular the resource supply of the plants. We tested this through a greenhouse

\section{Material and Methods}

\section{Plant material}


116 Reynoutria japonica and its hybrid Reynoutria $\times$ bohemica are large perennial forbs from the

117 Polygonaceae family that have become invasive in riparian and ruderal habitats in the

118 temperate regions of Europe and North America. They are clonal plants with extensive

119 rhizome networks, and in their invasive range they often form dense monoclonal stands and

120 become extremely dominant (Aguilera et al., 2010). In our experiment, we used plant material

121 from a live collection of knotweed clones that had originally been collected across seven

122 regions in Switzerland and Germany (Krebs et al., 2010) and that had been cultivated in a

123 common garden for several years. We used rhizome cuttings from $20 R$. japonica clones and

$12413 R . \times$ bohemica clones, with approximately ten rhizome fragments, each containing two

125 nodes and thus one intact internode, from each clone. After removal of all fine roots, the

126 rhizome fragments were surface-sterilized using the method described by (Huang et al.,

127 2014).

128

Endophyte material

130 We worked with the endophyte Serendipita herbamans (DSM 27534), a member of the order

131 Sebacinales, whose discovery and isolation was described in (Riess et al., 2014). Prior to the

132 experiment, we grew the endophyte for 14 days in Petri dishes with MEA medium containing

$1332 \%$ malt extract and $1.5 \%$ agar at $20^{\circ} \mathrm{C}$ in the dark. We then used $5 \mathrm{~mm}$ plugs from these

134 plates, containing media and mycelia, to inoculate $0.5 \mathrm{~L}$ Erlenmeyer flasks with $250 \mathrm{ml}$ malt

135 extract (2.0\%) liquid medium. The inoculated flasks were incubated in the dark on a rotary

136 shaker $(47-52 \mathrm{rpm})$ at $20^{\circ} \mathrm{C}$. After two weeks of incubation, the resulting mycelium was

137 separated from the media and washed five times with sterile distilled water.

138

139

Experimental set-up 
140 We set up a greenhouse experiment in which we tested the effects of endophyte inoculation

141 on knotweed growth in four different environments: control, drought, low nutrients and shade.

142 Except for the control environment, all conditions were expected to be stressful for the plants.

143 We planted individual rhizome fragments $3 \mathrm{~cm}$ deep in $1.5 \mathrm{~L}$ pots filled with a 1:3 mixture of

144 low-nutrient field soil and sand (Sand- und Kieswerk Bischoff, Rottenburg, Germany). Prior

145 to planting, we measured the length and diameter of each rhizome fragment. All pots were

146 placed on individual saucers and watered as needed. After two weeks, when all aboveground

147 shoots had appeared, we inoculated half of the pots with $0.5 \mathrm{~g}$ of fresh $S$. herbamans

148 mycelium which were applied in small pits close to the center of the rhizomes (Fig.1A). For

149 non-inoculated plants we also created the same pits and applied a similar volume of distilled

150 water. Another two weeks after the inoculation, we started the environmental treatments. In

151 the shade treatment, the plants were covered individually with shading mesh bags that

152 reduced light levels to approximately $20 \%$. The low-nutrient plants did not receive any

153 fertilizer throughout the experiment, whereas all others received 7:3:6 N:P:K fertilizer (b1

154 Universal-Flüssigdünger, toom Baumarkt $\mathrm{GmbH}$ ) equivalent to $150 \mathrm{~kg} \mathrm{~N} / \mathrm{ha}$ distributed

155 across 15 applications at seven-day intervals during the stress treatments. The drought plants

156 generally received only a third of the regular watering amount and, in contrast to all other

157 plants, regularly showed signs of wilting (loss of turgor). A total of 288 plants (160 R.

158 japonica and $128 R$. x bohemica) were randomly assigned to the eight treatment combinations

159 (four environmental conditions, with or without endophytes), with approximately equal

160 representation of the two taxa in each treatment. Throughout the experiment, the plants were

161 grown in a climate-controlled greenhouse, in a completely randomized order, with

162 supplemental lighting at a $14: 10 \mathrm{~h}$ light:dark cycle at $20^{\circ} \mathrm{C} / 18^{\circ} \mathrm{C}$. 
16515 weeks after the start of the treatments, we measured leaf chlorophyll content on four leaves

166 per plant using a handheld chlorophyll meter (SPAD 502Plus, Konica Minolta, Osaka, Japan).

167 We then removed all leaves from the stem, measured their area with a LI-3100C leaf area

168 meter (LI-COR Environmental, Lincoln, Nebraska, USA) and dried the leaves and stems of

169 each plant separately at $80 \square$ for 3 days. We used the leaf area and leaf dry mass to calculate

170 the specific leaf area (SLA) of each plant, and we combined the leaf and stem dry mass to

171 total aboveground biomass. Finally, we carefully washed the roots of each plant and took 10

172 fine root samples from different parts of the root system that we mixed and immediately

173 stored at $-20^{\circ} \mathrm{C}$ for subsequent DNA extraction, or placed in fixing solution $(0.15 \%$ (wt/vol)

174 trichloroacetic acid in 4:1 (vol/vol) ethanol/chloroform) for microscopy.

177 We assessed the fungal colonization of knotweed roots qualitatively through fluorescence

178 microscopy and quantitatively through qPCR. Both analyses were done for randomly selected

179 subsets of the plants. For the microscopy, we collected roots from three pots of each treatment

180 by species combination, including both inoculated and non-inoculated samples (altogether 16

181 x $3=48$ samples). The root samples were stained with Wheat Germ Agglutinin-Alexa Fluor

182488 (WGA488; Thermo Fisher, Waltham MA, USA), which specifically stains fungal cell

183 walls. The staining procedure was as described in (Deshmukh et al., 2006), and the images

184 were recorded on a Leica TCS SP5 2 confocal microscope using the bright field channel and a

185 GFP filter set for detection of WGA488.

186 For the qPCR, we analysed 10 plants from each treatment by species combination, i.e. a

187 total of 80 plants, with three replicates of non-inoculated plants and seven replicates for

188 inoculated plants. We ground fine roots to a fine powder in liquid nitrogen using a sterile

189 mortar and pestle, and we used $500 \mathrm{mg}$ of this material to isolate DNA using the DNAeasy 
190 Plant Mini Kit (Qiagen, Hilden, Germany). The relative amounts of S. herbamans DNA in the

191 samples were determined through qPCR reactions with a S. herbamans-specific and a

192 Reynoutria-specific primer pair. The S. herbamans-specific primer pair SerhaITS binds to the

193 ITS region of the 5.8 S rDNA sequence of S. herbamans (SerhaITSfw199: 5'-

194 AGCCTTGTGCGGTAAAGCGA-3', SerhaITSrev199: 5’'-

195 TGTATTCCGGCACCTTAACCTC-3'). The Reynoutria-specific primer pair FallCHS binds

196 to the genomic DNA of the Chalcone synthase gene EF090266.2 of Fallopia japonica (now

197 Reynoutria japonica) (FallCHSfwd: 5'-GGAGATGCGTGTATATTCTT-3', FallCHSrev: 5'-

198 CCAAAGATGAAGCCATGTAG-3'. The PCR primers were designed using Primer-BLAST

199 (Ye et al. 2012). For the PCR amplification we ran real-time PCRs on a Biorad CFX96

200 Thermocycler (BioRad, Hercules CA, USA) using the ABsolute SYBR Capillary Mix

201 (Thermo Fisher, Waltham MA, USA) in a final volume of $20 \mu \mathrm{l}$, and the following cycler

202 programmes: $95^{\circ} \mathrm{C}$ for 15 min followed by 45 cycles of $95^{\circ} \mathrm{C}$ for $15 \mathrm{~s}, 55^{\circ} \mathrm{C}$ for $20 \mathrm{~s}$ and $72^{\circ}$

$203 \mathrm{C}$ for $20 \mathrm{~s}$ for the FallCHS primer pair, and $95^{\circ} \mathrm{C}$ for $15 \mathrm{~min}$ followed by 45 cycles of $95^{\circ} \mathrm{C}$

204 for $15 \mathrm{~s}, 60^{\circ} \mathrm{C}$ for $15 \mathrm{~s}$ and $72^{\circ} \mathrm{C}$ for $10 \mathrm{~s}$ for the Serha199 primer pair. To calculate relative

205 amounts of S. herbamans DNA, we used the $2^{\text {-deltaCt }}$ method (Livak \& Schmittgen, 2001)

206 using the raw threshold cycle $(\mathrm{Ct})$ values determined for the S. herbamans- and the

207 Reynoutria-specific primer pairs.

208

209 Statistical analyses

210 To test for species differences in, and effects of environmental conditions on endophyte

211 colonization, we analysed the relative $S$. herbamans densities, as determined by qPCR, with a

212 linear model that included the effects of Reynoutria species, environmental treatment, and

213 their interaction as fixed factors. We analysed knotweed responses to endophytes and

214 environments with regard to three variables: aboveground biomass, leaf chlorophyll content, 
215 and specific leaf area. For each response variable we fitted a linear mixed model with fungal

216 inoculation, environmental treatments, knotweed species, and their interactions included as

217 fixed factors, and clone identity included as random factor. To account for possible influences

218 of initial size differences, we included the volume of the planted rhizome as a covariate in all

219 three analyses. Prior to the analyses, the biomass and specific leaf area data were log-

220 transformed to achieve homoscedasticity. All linear models were fitted with the lmer function

221 in the lme4 package (Bates et al., 2015) in R (R Core Team, 2018). We used the effects (Fox,

222 2003) and ggplot2 (Wickham, 2009) packages to visualize results.

223

224 Results

225 The experimental inoculation of knotweed plants with Serendipita herbamans was successful,

226 but relative colonization rates were strongly environment-dependent (Fig. 1C; main effect of

227 environmental treatment in the linear model: $F=27.12, P<0.001)$. While there were hardly

228 any fungi present in the non-inoculated samples, and the average relative colonization levels

229 remained low in the inoculated control and drought treatments, colonization increased four-

230 fold and eight-fold, respectively, under shaded and low nutrient conditions (Fig. 1C). There

231 were no differences among the two Reynoutria species in terms of relative fungal colonisation

232 ( $F<1$ and $P>0.5$ for species main effect and species $\mathrm{x}$ treatment interaction). The

233 colonization of Reynoutria roots by S. herbamans was confirmed by fluorescence

234 microscopy. In all root samples from inoculated plants we detected hyphal structures typical

235 for $S$. herbamans on root surfaces, between the outer cell layers, and inside of some cortical

236 root cells (Fig. 1B). We also observed some hyphal structures in the roots of non-inoculated

237 plants.

238 As expected, the stress treatments in our experiment strongly impacted the growth of

239 knotweed (Table 1, Fig. 2). Compared to control plants, the biomass was reduced in all three 
240 stress treatments, but particularly strongly under low-nutrient conditions. There were also

241 strong treatment effects on chlorophyll content and SLA, with a particularly low chlorophyll

242 content at low nutrient availability, and the highest SLA under shaded conditions (Fig. 2).

243 There were also differences between the two knotweed taxa (Table 1). The hybrid Reynoutria

$244 \times$ bohemica was generally larger (+25\%) and had a higher SLA (+ 5\%) than $R$. japonica, and

245 its biomass was less sensitive to drought and shading than that of $R$. japonica (Fig. S1).

246 Inoculation with Serendipita herbamans influenced the growth of the knotweed plants,

247 but again in a strongly treatment-dependent manner, with significant fungus by treatment

248 interactions (but no fungus main effects) for aboveground biomass and chlorophyll content,

249 and a marginally significant interaction for SLA (Table 1, Fig. 2). Under low-nutrient

250 conditions, addition of the endophyte increased knotweed biomass by $15 \%$, but decreased it

251 by $10 \%$ in the shade, or had no effect at all under drought or control conditions. Similarly,

252 endophyte inoculation increased chlorophyll content by $13 \%$ under low nutrients but

253 decreased it by $5 \%$ under shaded conditions, and had no significant effects in the other two

254 treatments. Finally, the SLA was positively affected in the shade but negatively under low-

255 nutrient conditions, with no effects under drought or control conditions.

\section{Discussion}

258 Plant-microbe interactions play an important role in natural ecosystems (Bever et al., 2012;

259 Klironomos, 2002; van der Putten et al., 2013). However, the ecological function of

260 endophytic microbes that live within plants is so far little understood. In this study we show

261 that the fungal root endophyte Serendipita herbamans can rapidly colonize invasive knotweed

262 (Reynoutria ssp.) and influence its growth, with detrimental effects in the shade but

263 promotion of growth under low-nutrient conditions. Our study thus demonstrates that this 
264 widespread endophyte interacts with an important invasive plant, and it also highlights the

265 environment-dependency of plant-endophyte interactions.

266

267

Endophyte colonization

268 Compared to previous studies on plant-Sebacinales interactions, our experiment had a rather

269 realistic set-up, with fungi inoculated into a non-sterile natural soil that presumably already

270 contained a microbial community. Microscopy and qPCR confirmed that our inoculations

271 were successful and that $S$. herbamans was able to colonize knotweed plants, which confirms

272 field observations in the Tübingen area where Sebacinales including S. herbamans are

273 frequent endophytes of invasive knotweed populations (Table S1). This is not surprising,

274 given the broad host range of Serendipita herbamans, and of Sebacinales in general, which

275 also includes native Polygonaceae (Garnica et al., 2013; Riess et al., 2014). Although exotic

276 species are known to lose specialised biotic interactions, they often interact with generalist

277 enemies and mutualists in the introduced range (Mitchell \& Power, 2003; Richardson et al.,

278 2007; van Kleunen et al., 2018). However, so far we do not know how novel the interaction

279 between knotweed and S. herbamans really is because there are no data on endophyte

280 diversity from the native East Asian range.

281 We found that the relative colonization of knotweed plants by $S$. herbamans was

282 generally much stronger under low-nutrients or shade conditions than under control or

283 drought conditions. Thus, Reynoutria plants appear to actively regulate their interactions with

$284 S$. herbamans in an environment-specific fashion. It is known that plants can control fungal

285 colonization, e.g. through the production of defense compounds or secondary metabolites

286 inhibiting microbial growth (Zipfel \& Oldroyd, 2017), or by diverting more carbohydrates to

287 fungal symbionts (Carbonnel \& Gutjahr, 2014; Martin et al., 2017). This has also been shown

288 for the closely related $S$. indica which interferes with the immune system of host plants 
289 (Jacobs et al., 2011) and influences sugar concentrations in their roots (Opitz et al., 2021).

290 The functional and adaptive explanation for this is usually that plant benefits from

291 interactions with fungi are environment-dependent, and therefore plants stimulate or restrict

292 fungal access depending on these benefits. For instance, mycorrhizal colonisation is often

293 triggered by low-nutrient conditions (Bueno de Mesquita et al., 2018). We also found that

294 relative fungal colonization was highest under low-nutrient conditions, which is in line with

295 the idea that $S$. herbamans improves the nutrition of Reynoutria plants. It is less clear why

296 relative colonization was also increased in shaded plants because these should have been

297 mainly carbon-limited, and under such conditions plant-microbe interactions often turn

298 parasitic, as has been shown e.g. for interactions with mycorrhiza or rhizobia (Ballhorn et al.,

299 2016; Lau et al., 2012).

300 We also detected S. herbamans in some non-inoculated plants. The sources of this could

301 be external, e.g. fungi spores present in the potting soil, or splash dispersal from adjacent pots.

302 However, the most likely explanation seems that $S$. herbamans was already present in some of

303 the planted rhizomes. We know that some invasive knotweed populations are naturally

304 colonized by S. herbamans, and we therefore cannot rule out that some surface-sterilized

305 rhizomes still harboured the fungus.

306

307 Endophyte effects on plant growth

308 The inoculated $S$. herbamans fungi not only successfully colonized the knotweed plants in our

309 experiment, but they also significantly impacted their growth. The magnitude and direction of

310 these effects were strongly environment-dependent. Under benign or drought conditions,

311 endophyte effects on plants were small and non-significant, whereas under low-nutrient

312 conditions inoculation had strong positive effects, and under shade conditions strong negative 
313 effects on knotweed performance. Similar context-dependent effects of endophytes have been

314 found in other study systems (Davitt et al., 2010; Laitinen et al., 2016; Shaffer et al., 2018).

315 Low-nutrient conditions greatly reduced knotweed biomass, and here relative $S$.

316 herbamans colonization was strong and the fungus increased plant growth. This suggests an

317 active promotion of endophyte access by the plants because the fungi improve plant nutrition

318 under these conditions. The observed increase of leaf chlorophyll content, which strongly

319 correlates with leaf nitrogen content (Evans, 1989), supports this idea. We know that $S$.

320 herbamans improves plant growth under lab conditions (Riess et al., 2014), and that the

321 closely related Serendipita indica can improve the nutrient acquisition and growth of many

322 plant species (Achatz et al., 2010; Barazani et al., 2005; Giauque et al., 2019; Varma et al.,

323 1999; Waller et al., 2005). Thus, it seems very likely that S. herbamans also improved the

324 nutrition, and as a consequence biomass growth, of invasive knotweed in our experiment.

325 Under shade conditions, the effects of $S$. herbamans were reversed, and inoculation

326 negatively affected knotweed biomass as well as leaf chlorophyll content, suggesting that

327 under these conditions the fungus indeed turned parasitic and compromised plant nutrition.

328 Similar shifts in the directions of plant-microbe interactions have been observed in other

329 studies (Ballhorn et al., 2016; Lau et al., 2012), and the likely explanation is that the typical

330 'trade logic' of mutualistic plant-microbe interactions - microbes receive photosynthates in

331 exchange for improved nutrient uptake - only works where soil nutrients are limiting, but

332 under carbon-limited shade conditions, it does not.

333 In the control and drought treatments, colonization and growth effects of endophytes

334 were very low, indicating that under these conditions the host plants limited fungi access,

335 similar to what is known from plant-mycorrhiza interactions (Averill et al., 2019; Carbonnel

$336 \&$ Gutjahr, 2014). For the drought treatment, with episodes of plant wilting, it is also possible

337 that the spread of fungi was simply limited by the lack of moisture. 
Our results that $S$. herbamans can promote or weaken knotweed growth depending on

339 environmental context raises intriguing questions about the habitat preferences of invasive

340 knotweeds. Across their invasive range in Europe and North America, the species mostly

341 thrive in open and nutrient-rich habitats, and benefit in particular from fluctuating nutrient

342 supply (Parepa, Fischer, et al., 2013), but they rarely spread under closed canopy (Beerling,

343 1991; Pyšek et al., 2009). It is possible that interactions with S. herbamans or other microbes

344 contribute to these habitat preferences, by facilitating nutrient uptake in open habitats but

345 limiting knotweed under shaded conditions. Further research - in particular field experiments

346 - is needed to test these hypotheses.

347

348 Conclusions

349 Our study demonstrates that the common fungal endophyte Serendipita herbamans can

350 rapidly colonize fine roots of invasive knotweed and influence its growth both positively or

351 negatively, depending on the environmental context. As S. herbamans is present in at least

352 some invasive knotweed populations, the fungus could play a role in the growth and success

353 of knotweed in some invaded habitats. However, understanding the true significance of this

354 plant-fungus interaction requires further data, because ecological communities are of course

355 more complex than our experimental set-up. In its natural habitat, invasive knotweed also

356 interacts with competitors, herbivores and other enemies and mutualists, and some of these

357 might be interacting with $S$. herbamans, too. Thus, the next step should be multi-species

358 experiments, in the field or using mesocosm approaches, that evaluate the impact of $S$.

359 herbamans on knotweed and other plants in a community context.

360

361 Acknowledgments 
362 We thank Christiane Karasch-Wittmann, Sabine Silberhorn, Julia Rafalski, Mirjam Rieger,

363 Mohamed Osman, and several student helpers for their assistance with the greenhouse

364 experiment and/or molecular work. This project was supported by the German Research

365 Foundation (DFG grant BO 3241/8-1 to OB), and by a scholarship of the China Scholarship

366 Council (grant no. 201504910498) to Zhiyong Liao.

368 Authors' contributions

369 SG, MP and OB conceived and designed the experiment, SG, ZL and SH carried out the

370 experiment, FW performed microscopy and qPCR analyses, MP and OB analyzed the data,

371 SG, FW, MP and OB drafted the manuscript, and all authors contributed to its revision.

372

373 Data availability

374 All data from our experiment will be made available through Dryad. The sequencing data

375 from the supplement are stored at GeneBank under MZ650923 - MZ651047.

376

377

378

379

380

381

382

383

384

385

386

\section{References}

Achatz, B., von Rüden, S., Andrade, D., Neumann, E., Pons-Kühnemann, J., Kogel, K.-H., Franken, P., \& Waller, F. (2010). Root colonization by Piriformospora indica enhances grain yield in barley under diverse nutrient regimes by accelerating plant development. Plant and Soil, 333(1), 59-70.

Afkhami, M. E., \& Strauss, S. Y. (2016). Native fungal endophytes suppress an exotic dominant and increase plant diversity over small and large spatial scales. Ecology, 97(5), 1159-1169.

Aguilar-Trigueros, C. A., \& Rillig, M. C. (2016). Effect of different root endophytic fungi on plant community structure in experimental microcosms. Ecology and Evolution, 6(22), 
8149-8158.

388

389

390

391

392

393

394

395

396

397

398

402

403

404

405

406

407

408

409

410

411

Aguilera, A. G., Alpert, P., Dukes, J. S., \& Harrington, R. (2010). Impacts of the invasive plant Fallopia japonica (Houtt.) on plant communities and ecosystem processes. Biological Invasions, 12(5), 1243-1252.

Aschehoug, E. T., Callaway, R. M., Newcombe, G., Tharayil, N., \& Chen, S. (2014). Fungal endophyte increases the allelopathic effects of an invasive forb. Oecologia, 175(1), 285291.

Aschehoug, E. T., Metlen, K. L., Callaway, R. M., \& Newcombe, G. (2012). Fungal endophytes directly increase the competitive effects of an invasive forb. Ecology, 93(1), $3-8$.

Averill, C., Bhatnagar, J. M., Dietze, M. C., Pearse, W. D., \& Kivlin, S. N. (2019). Global imprint of mycorrhizal fungi on whole-plant nutrient economics. Proceedings of the National Academy of Sciences of the United States of America, 116(46), 23163-23168.

Ballhorn, D. J., Schädler, M., Elias, J. D., Millar, J. A., \& Kautz, S. (2016). Friend or FoeLight Availability Determines the Relationship between Mycorrhizal Fungi, Rhizobia and Lima Bean (Phaseolus lunatus L.). PloS ONE, 11(5), e0154116.

Barazani, O., Benderoth, M., Groten, K., Kuhlemeier, C., \& Baldwin, I. T. (2005). Piriformospora indica and Sebacina vermifera increase growth performance at the expense of herbivore resistance in Nicotiana attenuata. Oecologia, 146(2), 234-243.

Bates, D., Mächler, M., Bolker, B., \& Walker, S. (2015). Fitting linear mixed-effects models using lme4. Journal of Statistical Software, 67(1), 1-48.

Beerling, D. J. (1991). The effect of Riparian land use on the occurrence and abundance of Japanese knotweed Reynoutria japonica on selected rivers in South Wales. Biological Conservation, 55(3), 329-337.

Behie, S. W., \& Bidochka, M. J. (2014). Nutrient transfer in plant-fungal symbioses. Trends 
in Plant Science, 19(11), 734-740.

413 Bever, J. D., Platt, T. G., \& Morton, E. R. (2012). Microbial population and community

414 dynamics on plant roots and their feedbacks on plant communities. Annual Review of

415 Microbiology, 66, 265-283.

416 Bueno de Mesquita, C. P., Sartwell, S. A., Ordemann, E. V., Porazinska, D. L., Farrer, E. C.,

417 King, A. J., Spasojevic, M. J., Smith, J. G., Suding, K. N., \& Schmidt, S. K. (2018).

418 Patterns of root colonization by arbuscular mycorrhizal fungi and dark septate

419 endophytes across a mostly-unvegetated, high-elevation landscape. Fungal Ecology, 36, $420 \quad 63-74$.

421 Callaway, R. M., Bedmar, E. J., Reinhart, K. O., Silvan, C. G., \& Klironomos, J. (2011).

422 Effects of soil biota from different ranges on Robinia invasion: acquiring mutualists and 423 escaping pathogens. Ecology, 92(5), 1027-1035.

424 Callaway, R. M., Thelen, G. C., Rodriguez, A., \& Holben, W. E. (2004). Soil biota and exotic 425 plant invasion. Nature, 427(6976), 731-733.

426 Carbonnel, S., \& Gutjahr, C. (2014). Control of arbuscular mycorrhiza development by $427 \quad$ nutrient signals. Frontiers in Plant Science, 5, 462.

428 Clay, K., \& Holah, J. (1999). Fungal endophyte symbiosis and plant diversity in successional 429 fields. Science, 285(5434), 1742-1745.

430 Clay, K., Shearin, Z. R. C., Bourke, K. A., Bickford, W. A., \& Kowalski, K. P. (2016).

431 Diversity of fungal endophytes in non-native Phragmites australis in the Great Lakes.

$432 \quad$ Biological Invasions, 18(9), 2703-2716.

433 Cosme, M., Lu, J., Erb, M., Stout, M. J., Franken, P., \& Wurst, S. (2016). A fungal endophyte

434 helps plants to tolerate root herbivory through changes in gibberellin and jasmonate 435 signaling. New Phytologist, 211(3), 1065-1076.

436 Davitt, A. J., Stansberry, M., \& Rudgers, J. A. (2010). Do the costs and benefits of fungal 

endophyte symbiosis vary with light availability? New Phytologist, 188(3), 824-834.

438 Dawson, W., \& Schrama, M. (2016). Identifying the role of soil microbes in plant invasions. $439 \quad$ Journal of Ecology, 104(5), 1211-1218.

440 Deshmukh, S., Hückelhoven, R., Schäfer, P., Imani, J., Sharma, M., Weiss, M., Waller, F., \& 441 Kogel, K.-H. (2006). The root endophytic fungus Piriformospora indica requires host 442 cell death for proliferation during mutualistic symbiosis with barley. Proceedings of the $443 \quad$ National Academy of Sciences of the United States of America, 103(49), 18450-18457.

444 Evans, J. R. (1989). Photosynthesis and nitrogen relationships in leaves of C3 plants.

$445 \quad$ Oecologia, 78(1), 9-19.

446 Fox, J. (2003). Effect displays in R for generalised linear models. Journal of Statistical $447 \quad$ Software, $8(15), 1-27$.

448 Garnica, S., Riess, K., Bauer, R., Oberwinkler, F., \& Weiß, M. (2013). Phylogenetic diversity 449 and structure of sebacinoid fungi associated with plant communities along an altitudinal $450 \quad$ gradient. FEMS Microbiology Ecology, 83(2), 265-278.

451 Garnica, S., Riess, K., Schön, M. E., Oberwinkler, F., \& Setaro, S. D. (2016). Divergence 452 times and phylogenetic patterns of Sebacinales, a highly diverse and widespread fungal $453 \quad$ lineage. PLoS ONE, 11(3), e0149531.

454 Gerber, E., Krebs, C., Murrell, C., Moretti, M., Rocklin, R., \& Schaffner, U. (2008). Exotic 455 invasive knotweeds (Fallopia spp.) negatively affect native plant and invertebrate 456 assemblages in European riparian habitats. Biological Conservation, 141(3), 646-654.

457 Giauque, H., Connor, E. W., \& Hawkes, C. V. (2019). Endophyte traits relevant to stress 458 tolerance, resource use and habitat of origin predict effects on host plants. New $459 \quad$ Phytologist, 221(4), 2239-2249.

460 Gill, S. S., Gill, R., Trivedi, D. K., Anjum, N. A., Sharma, K. K., Ansari, M. W., Ansari, A. 461 A., Johri, A. K., Prasad, R., Pereira, E., Varma, A., \& Tuteja, N. (2016). Piriformospora 
462

463

464

465

466

467

468

469

470

471

472

473

474

475

476

477

478

479

480

481

482

483

484

485

486

indica: potential and significance in plant stress tolerance. Frontiers in Microbiology, 7 , 332.

Hejda, M., Pyšek, P., \& Jarošík, V. (2009). Impact of invasive plants on the species richness, diversity and composition of invaded communities. Journal of Ecology, 97(3), 393-403.

Huang, B., Lin, H., Yan, C., Qiu, H., Qiu, L., \& Yu, R. (2014). Optimal inductive and cultural conditions of Polygonum multiflorum transgenic hairy roots mediated with Agrobacterium rhizogenes R1601 and an analysis of their anthraquinone constituents. Pharmacognosy Magazine, 10(37), 77-82.

Inderjit, \& van der Putten, W. H. (2010). Impacts of soil microbial communities on exotic plant invasions. Trends in Ecology \& Evolution, 25(9), 512-519.

Jacobs, S., Zechmann, B., Molitor, A., Trujillo, M., Petutschnig, E., Lipka, V., Kogel, K.-H., \& Schäfer, P. (2011). Broad-spectrum suppression of innate immunity is required for colonization of Arabidopsis roots by the fungus Piriformospora indica. Plant Physiology, 156(2), 726-740.

Kivlin, S. N., Emery, S. M., \& Rudgers, J. A. (2013). Fungal symbionts alter plant responses to global change. American Journal of Botany, 100(7), 1445-1457.

Klironomos, J. N. (2002). Feedback with soil biota contributes to plant rarity and invasiveness in communities. Nature, 417(6884), 67-70.

Krebs, C., Mahy, G., Matthies, D., Schaffner, U., Tiébré, M.-S., \& Bizoux, J.-P. (2010). Taxa distribution and RAPD markers indicate different origin and regional differentiation of hybrids in the invasive Fallopia complex in central-western Europe. Plant Biology, 12(1), 215-223.

Laitinen, R. K., Hellström, K. O., \& Wäli, P. R. (2016). Context-dependent outcomes of subarctic grass-endophyte symbiosis. Fungal Ecology, 23, 66-74.

Lau, J. A., Bowling, E. J., Gentry, L. E., Glasser, P. A., Monarch, E. A., Olesen, W. M., 
Waxmonsky, J., \& Young, R. T. (2012). Direct and interactive effects of light and nutrients on the legume-rhizobia mutualism. Acta Oecologica, 39, 80-86.

489

490

491

492

493

494

495

496

497

498

499

500

501

502

503

504

505

506

507

508

509

510

511

Livak, K. J., \& Schmittgen, T. D. (2001). Analysis of relative gene expression data using realtime quantitative PCR and the 2(-Delta Delta C(T)) method. Methods, 25(4), 402-408.

Mangla, S., \& Callaway, R. M. (2007). Exotic invasive plant accumulates native soil pathogens which inhibit native plants. Journal of Ecology, O(0), 071031082432001 - ???

Maron, J. L., Klironomos, J., Waller, L., \& Callaway, R. M. (2014). Invasive plants escape from suppressive soil biota at regional scales. Journal of Ecology, 102(1), 19-27.

Martin, F. M., Uroz, S., \& Barker, D. G. (2017). Ancestral alliances: Plant mutualistic symbioses with fungi and bacteria. Science, 356(6340). https://doi.org/10.1126/science.aad4501

Mayerhofer, M. S., Kernaghan, G., \& Harper, K. A. (2013). The effects of fungal root endophytes on plant growth: a meta-analysis. Mycorrhiza, 23(2), 119-128.

Meinhardt, K. A., \& Gehring, C. A. (2012). Disrupting mycorrhizal mutualisms: a potential mechanism by which exotic tamarisk outcompetes native cottonwoods. Ecological Applications: A Publication of the Ecological Society of America, 22(2), 532-549.

Mitchell, C. E., \& Power, A. G. (2003). Release of invasive plants from fungal and viral pathogens. Nature, 421(6923), 625-627.

Murrell, C., Gerber, E., Krebs, C., Parepa, M., Schaffner, U., \& Bossdorf, O. (2011). Invasive knotweed affects native plants through allelopathy. American Journal of Botany, 98(1), $38-43$.

Oberhofer, M., Güsewell, S., \& Leuchtmann, A. (2014). Effects of natural hybrid and nonhybrid Epichloë endophytes on the response of Hordelymus europaeus to drought stress. New Phytologist, 201(1), 242-253.

Omacini, M., Chaneton, E. J., Ghersa, C. M., \& Müller, C. B. (2001). Symbiotic fungal 
512 endophytes control insect host-parasite interaction webs. Nature, 409(6816), 78-81.

513 Opitz, M. W., Daneshkhah, R., Lorenz, C., Ludwig, R., Steinkellner, S., \& Wieczorek, K.

514 (2021). Serendipita indica changes host sugar and defense status in Arabidopsis thaliana:

$515 \quad$ cooperation or exploitation? Planta, 253(3), 74.

516 Parepa, M., Fischer, M., \& Bossdorf, O. (2013). Environmental variability promotes plant 517 invasion. Nature Communications, 4, 1604.

518 Parepa, M., Schaffner, U., \& Bossdorf, O. (2013). Help from under ground: soil biota

519 facilitate knotweed invasion. Ecosphere, 4(2), art31.

520 Pyšek, P., Jarošík, V., Pergl, J., Randall, R., Chytrý, M., Kühn, I., Tichý, L., Danihelka, J., 521 Chrtek jun, J., \& Sádlo, J. (2009). The global invasion success of Central European 522 plants is related to distribution characteristics in their native range and species traits. 523 Diversity \& Distributions, 15(5), 891-903.

524 R Core Team. (2018). R: A language and environment for statistical computing. R

$525 \quad$ Foundation for Statistical Computing.

526 Reinhart, K. O., Packer, A., Van der Putten, W. H., \& Clay, K. (2003). Plant-soil biota

527 interactions and spatial distribution of black cherry in its native and invasive ranges.

$528 \quad$ Ecology Letters, 6(12), 1046-1050.

529 Rho, H., Hsieh, M., Kandel, S. L., Cantillo, J., Doty, S. L., \& Kim, S.-H. (2018). Do

530 Endophytes Promote Growth of Host Plants Under Stress? A Meta-Analysis on Plant

$531 \quad$ Stress Mitigation by Endophytes. Microbial Ecology, 75(2), 407-418.

532 Richardson, D. M., Allsopp, N., D’antonio, C. M., Milton, S. J., \& Rejmánek, M. (2007).

533 Plant invasions - the role of mutualisms. Biological Reviews, 75(1), 65-93.

534 Riess, K., Oberwinkler, F., Bauer, R., \& Garnica, S. (2014). Communities of endophytic

535 sebacinales associated with roots of herbaceous plants in agricultural and grassland

536 ecosystems are dominated by Serendipita herbamans sp. nov. PLoS ONE, 9(4), e94676. 
537 Rodriguez, R. J., Henson, J., Van Volkenburgh, E., Hoy, M., Wright, L., Beckwith, F., Kim,

538 Y.-O., \& Redman, R. S. (2008). Stress tolerance in plants via habitat-adapted symbiosis. $539 \quad$ The ISME Journal, 2(4), 404-416.

540 Rodriguez, R. J., White, J. F., Jr, Arnold, A. E., \& Redman, R. S. (2009). Fungal endophytes:

541 diversity and functional roles. New Phytologist, 182(2), 314-330.

542 Rudgers, J. A., Koslow, J. M., \& Clay, K. (2004). Endophytic fungi alter relationships

543 between diversity and ecosystem properties. Ecology Letters, 7(1), 42-51.

544 Rudgers, J. A., Mattingly, W. B., \& Koslow, J. M. (2005). Mutualistic fungus promotes plant

545 invasion into diverse communities. Oecologia, 144(3), 463-471.

546 Shaffer, J. P., Zalamea, P.-C., Sarmiento, C., Gallery, Rachel E., Dalling, J. W., Davis, A. S.,

547 Baltrus, D. A., \& Arnold, A. E. (2018). Context-dependent and variable effects of

548 endohyphal bacteria on interactions between fungi and seeds. Fungal Ecology, 36, 117-

$549 \quad 127$.

550 Shipunov, A., Newcombe, G., Raghavendra, A. K. H., \& Anderson, C. L. (2008). Hidden

551 diversity of endophytic fungi in an invasive plant. American Journal of Botany, 95(9),

$552 \quad 1096-1108$.

553 Siemens, T. J., \& Blossey, B. (2007). An evaluation of mechanisms preventing growth and

554 survival of two native species in invasive Bohemian knotweed (Fallopia $\mathrm{x}$ bohemica,

555 Polygonaceae). American Journal of Botany, 94(5), 776-783.

556 Stinson, K. A., Campbell, S. A., Powell, J. R., Wolfe, B. E., Callaway, R. M., Thelen, G. C.,

557 Hallett, S. G., Prati, D., \& Klironomos, J. N. (2006). Invasive plant suppresses the

558 growth of native tree seedlings by disrupting belowground mutualisms. PLoS Biology,

$559 \quad 4(5), \mathrm{e} 140$.

560 van der Putten, W. H., Bardgett, R. D., Bever, J. D., Bezemer, T. M., Casper, B. B., Fukami,

561 T., Kardol, P., Klironomos, J. N., Kulmatiski, A., Schweitzer, J. A., Suding, K. N., Van 
de Voorde, T. F. J., \& Wardle, D. A. (2013). Plant-soil feedbacks: the past, the present and future challenges. Journal of Ecology, 101(2), 265-276.

564

van Kleunen, M., Bossdorf, O., \& Dawson, W. (2018). The Ecology and Evolution of Alien

565 Plants. Annual Review of Ecology, Evolution, and Systematics, 49(1), 25-47.

566

Varma, A., Savita, V., Sudha, Sahay, N., Butehorn, B., \& Franken, P. (1999). Piriformospora

567

568 indica, a cultivable plant-growth-promoting root endophyte. Applied and Environmental Microbiology, 65(6), 2741-2744.

570

Waller, F., Achatz, B., Baltruschat, H., Fodor, J., Becker, K., Fischer, M., Heier, T., Hückelhoven, R., Neumann, C., von Wettstein, D., Franken, P., \& Kogel, K.-H. (2005). The endophytic fungus Piriformospora indica reprograms barley to salt-stress tolerance, disease resistance, and higher yield. Proceedings of the National Academy of Sciences of

574 Weiss, M., Sýkorová, Z., Garnica, S., Riess, K., Martos, F., Krause, C., Oberwinkler, F., 575 Bauer, R., \& Redecker, D. (2011). Sebacinales everywhere: previously overlooked $576 \quad$ ubiquitous fungal endophytes. PLoS ONE, 6(2), e16793.

577 Wickham, H. (2009). ggplot2: Elegant Graphics for Data Analysis. Springer-Verlag.

578 Ye, J., Coulouris, G., Zaretskaya, I., Cutcutache, I., Rozen, S., \& Madden, T. L. (2012)

579 Primer-BLAST: a tool to design target-specific primers for polymerase chain reaction. $580 \quad$ BMC Bioinformatics, 13, 134.

581 Zipfel, C., \& Oldroyd, G. E. D. (2017). Plant signalling in symbiosis and immunity. Nature, $582 \quad 543(7645), 328-336$. 
Table 1. Analysis of variance testing the effects of inoculation with Serendipita herbamans, stress treatment and knotweed species (Reynoutria japonica or $R . \times$ bohemica), and their interactions, on the performance of invasive knotweed. Each linear mixed model additionally included the volume of the planted rhizome as a covariate, as well as knotweed clone identity as a random variable. Significant $P$-values are in bold.

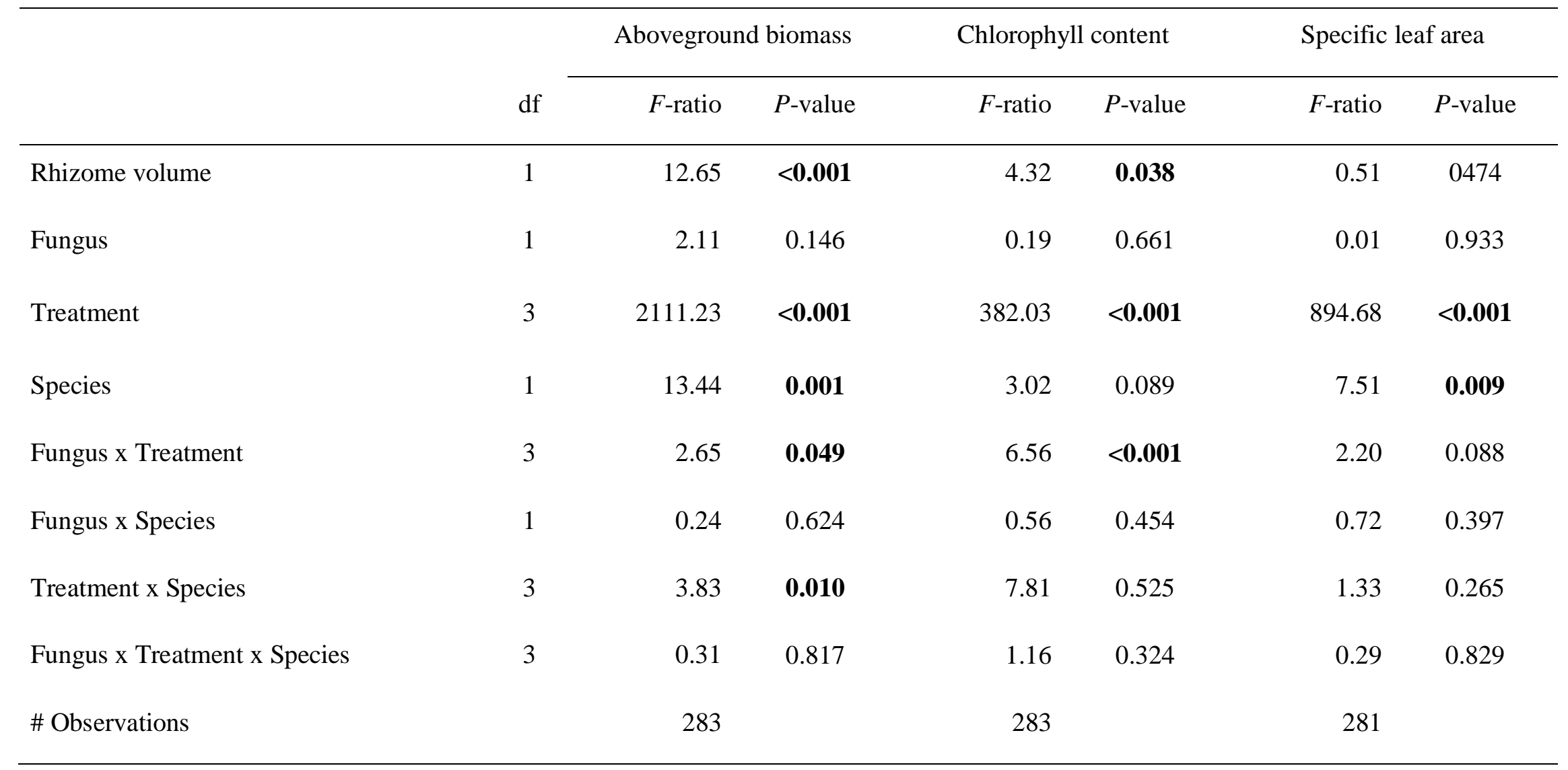



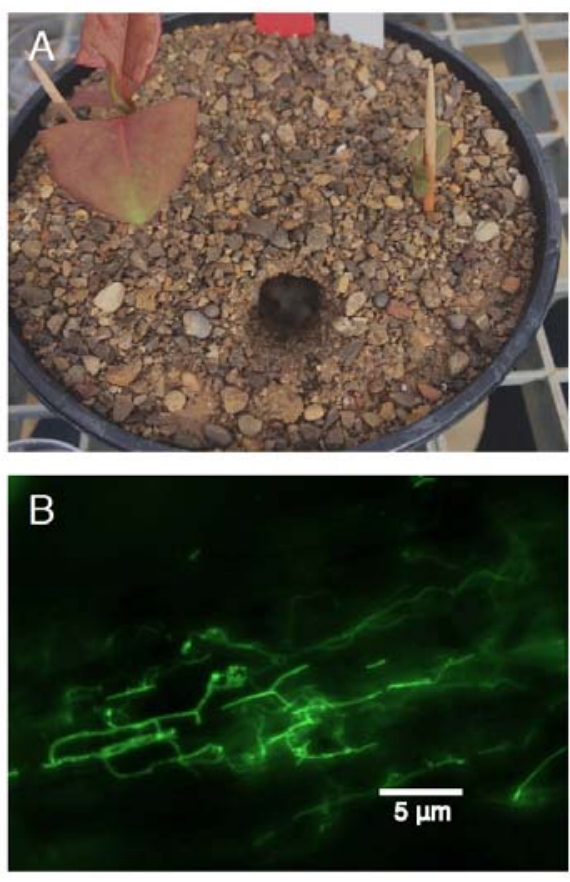

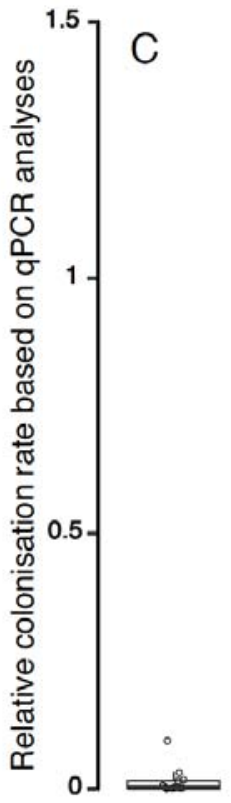

Non-inoculated

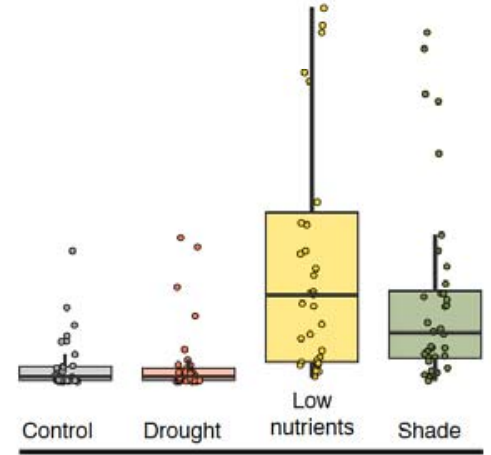

Inoculated

Figure 1. Experimental inoculation of Reynoutria plants with Serendipita herbamans, and the resulting fungal colonization. (A) An experimental pot right after inoculation, with freshly regenerated knotweed and the pit through which $S$. herbamans mycelium was added. (B) Fluorescence microscopic image of a root section of an inoculated Reynoutria plant, stained with WGA-AF 488. (C) Relative colonization rates of $S$. herbamans in the different experimental treatments, based on qPCR analyses. Points = individual observations; boxes = 25 th -75 th percentiles; thick horizontal lines $=$ medians; whiskers $=10$ th -90 th percentiles . 


\section{Biomass}

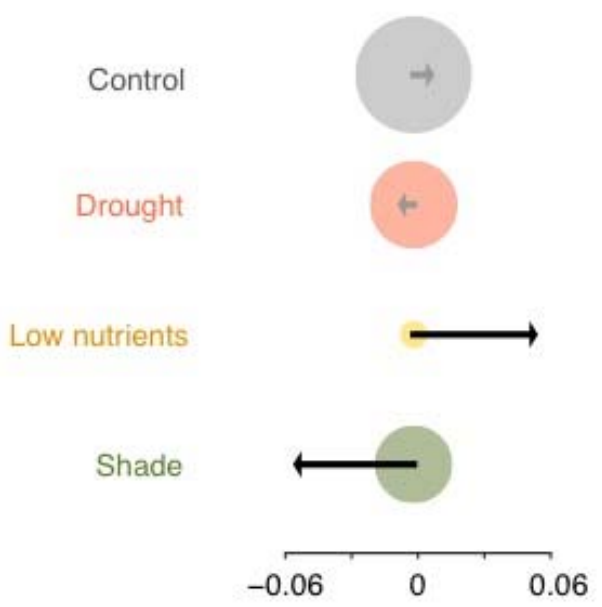

Specific leaf area
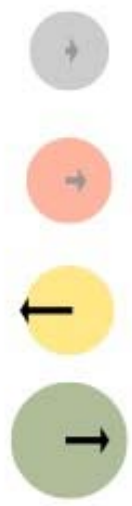

0.06

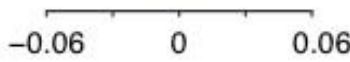

$-0.06$

Chlorophyll

Response to endophyte inoculation [log10 (with endophyte/without endophyte)]

Figure 2. Effects of inoculation with Serendipita herbamans on invasive knotweed aboveground biomass, leaf chlorophyll content, and specific leaf area under different types of stress and in control conditions. The disc areas are proportional to the estimated means without endophyte inoculation, and the arrows show the log-responses of plants to endophyte inoculation in each treatment. Black arrows represent significant endophyte effects within a particular treatment, as indicated by Tukey's HSD test, grey arrows are non-significant. 